\title{
Patrimoine et commun(s)
}

Une proximité incertaine

\section{Francesca Cominelli, Marie Cornu et Jean-Louis Tornatore}

\section{(2) OpenEdition}

\section{Journals}

Édition électronique

URL : https://journals.openedition.org/insituarss/589

DOI : 10.4000/insituarss.589

ISSN : 2680-4972

Éditeur

Ministère de la Culture

Référence électronique

Francesca Cominelli, Marie Cornu et Jean-Louis Tornatore, "Patrimoine et commun(s) », In Situ. Au regard des sciences sociales [En ligne], 2 | 2021, mis en ligne le 18 mars 2021, consulté le 23 janvier 2023. URL : http://journals.openedition.org/insituarss/589; DOI : https://doi.org/10.4000/insituarss. 589

Ce document a été généré automatiquement le 23 janvier 2023.

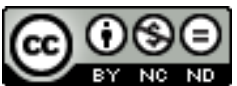

Creative Commons - Attribution - Pas d'Utilisation Commerciale - Pas de Modification 4.0 International - CC BY-NC-ND 4.0

https://creativecommons.org/licenses/by-nc-nd/4.0/ 


\title{
Patrimoine et commun(s)
}

\author{
Une proximité incertaine \\ Francesca Cominelli, Marie Cornu et Jean-Louis Tornatore
}

1 La question des « communs » est de plus en plus au cœur des projets de recherche, des politiques publiques, des discours politiques, des projets citoyens, des mouvements sociaux, des contestations collectives... et selon des perspectives, cette livraison en témoigne, qui ne sont certes pas convergentes. Si les travaux d'Elinor Ostrom ont joué un rôle crucial pour construire un courant de recherche dans ce domaine, la thématique des communs a acquis ces dernières années une importance majeure, ce que montre la diversité des sciences humaines qu'elle intéresse - à l'instar des coordonnatrices et du coordinateur de ce numéro, respectivement économiste, juriste et anthropologue,. Les publications de Benjamin Coriat (2015), Hervé Le Crosnier (2015), David Bollier (2014), Marie Cornu, Fabienne Orsi et Judith Rochfeld (2017) en France, ou encore les travaux de la Commission Rodotà (Rodotà 2013), de Ugo Mattei (2011) et de Daniela Festa (2016) en Italie ont permis d'élargir le champ de recherche aux communs de la connaissance et du numérique, et aussi d'étendre les perspectives d'application de cette notion à la compréhension de nos sociétés et à leur construction. Quand d'autres, celles en particulier de Pierre Dardot et Christian Laval (2014) ou de Pascal Nicolas Le Strat (2016), ont vu dans le commun, entendu comme principe politique portant des revendications contre l'ordre capitaliste et l'État entrepreneurial, le moyen d'engager leur transformation radicale.

2 Les communs se manifestent de différentes manières: une transformation des politiques publiques, une incitation à l'engagement citoyen, des formes de résistance et des inventions du quotidien appuyées sur une conception ouverte de la culture et sur de nouvelles alliances, une approche à l'éducation permanente, un renforcement des droits fondamentaux et culturels, des nouveaux modèles économiques, mais aussi culturels et sociaux. Les communs expriment la nécessité d'un esprit éveillé et critique face aux transformations accélérées des sociétés, face aux inquiétudes grandissantes exprimées dans un horizon d'effondrement, devant lesquelles s'affrontent d'un côté des nouvelles formes d'enclosures et de marchandisation, et de l'autre côté des pratiques alternatives, fondées sur le collectif, l'auto-organisation, la mise en commun, comme le 
montrent les expériences de Notre-Dame-des-Landes ou du Teatro Valle à Rome. Parce que les communs se retrouvent dans des domaines différents, incorporent des valeurs multiples, deviennent l'expression de conflits et revendications culturelles, sociales et politiques, on conçoit que leur définition ne soit pas consensuelle ${ }^{1}$. Se plaçant dans le sillage des travaux d'Elinor Ostrom, Benjamin Coriat en a dégagé les traits saillants : " Des ensembles de ressources en accès partagé et collectivement gouvernées au moyen d'une structure de gouvernance assurant une distribution des droits et des obligations entre les participants au commun (commoners ${ }^{2}$ ) et visant à l'exploitation ordonnée de la ressource, permettant sa reproduction dans le long terme " (2017). Cette définition générique d'une idée de vive actualité est loin d'épuiser la complexité de la réalité qu'elle désigne et qui, comme on pourra le lire, se révèle sous des termes différents : les biens communs, les communs, le commun, le commoning. Si cela n'était pas notre point de départ, on verra que notre questionnement sur le lien entre patrimoine et commun(s) peut s'insérer dans la compréhension politique de cette variation terminologique.

\section{Patrimoine et commun(s), un questionnement}

Confrontant patrimoine et commun(s), dans ce numéro, nous cherchons à comprendre quels pourraient en être les points de contact, quels ordres de relation établir entre ces deux catégories, les territoires qu'elles investissent et les pratiques qu'elles induisent. En quoi notamment l'outillage théorique des communs permettrait-il de mieux penser et définir le patrimoine ? Symétriquement, à quoi nous sert le patrimoine dès lors qu'il s'agit d'exprimer des revendications sociales, de comprendre ou construire les modes sur lesquels elles se structurent et se gouvernent, de creuser la question des responsabilités individuelles et collectives ${ }^{3}$ ? Le patrimoine serait-il un meilleur outil ou plus simplement un bon outil pour identifier et qualifier et organiser la complexité des relations qui se nouent autour de certaines choses, pour appréhender les formes de sociabilité que les communs instaurent et qui les instaurent? Et si leur relation n'est pas réciproque, ne doit-on pas se demander dans quelle mesure les communs permettent d'appréhender différemment la notion de patrimoine, et même s'ils n'invitent pas à la dépasser et à la périmer ?

Dès lors que dans les deux cas, il est question de ressources partagées en commun, le lien entre patrimoine et communs paraît tout naturellement s'imposer, du moins au premier abord. Dans les travaux sur les communs, les monuments historiques sont couramment pris en exemple. On peut évoquer François Ost (2003) lorsqu'il élabore la notion de transpropriation qui signale que la propriété ne peut se résumer à une relation exclusive entre le propriétaire et sa chose en ce qu'elle accueille aussi des valeurs et des intérêts collectifs ou encore Judith Rochfeld (2014) lorsqu'elle approfondit les modèles juridiques des communs. On peut bien admettre que le patrimoine a à voir avec les communs, en tant qu'ils sont sous-tendus par les idées de partage et de ressource collective. On entend, communément, qu'il renvoie à cet ensemble de choses qui appartiennent à tous, même si la formule relève du registre du symbolique et non du juridique (Wagener 2012). La notion de patrimoine commun, quand bien même elle est juridiquement peu chargée, paraît bien être à ce point de jonction, comme le soutient Meryem Deffairi (ce numéro). Elle court dans un certain nombre de textes pour qualifier l'eau, le territoire, la langue, le sous-sol archéologique, 
etc. La proximité avec les communs est encore plus marquée dans les développements récents qui déplacent la question patrimoniale vers la relation entre les personnes et les choses, en particulier celles qu'entretiennent les communautés avec ce qu'elles revendiquent comme leur patrimoine. Les textes internationaux assurent la promotion de ce nouveau "droit au patrimoine " selon l'expression de la Convention de Faro signée en 2005 dans le cadre du Conseil de l'Europe. L'approche est également portée par les conventions Unesco de dernière génération : la Convention pour la sauvegarde du patrimoine culturel immatériel de 2003 et la Convention sur la protection et la promotion de la diversité des expressions culturelles de 2005. Ainsi le cadre normatif international crée un espace pour la reconnaissance des communautés et de leur rôle actif dans l'identification, la création, la transmission et la sauvegarde du patrimoine. Cette sollicitation à l'implication des communautés est renforcée dans la mise en œuvre de ces textes juridiques au niveau national et peut inspirer des nouvelles pratiques de sauvegarde ${ }^{4}$. À cette démarche institutionnelle, s'ajoutent des expériences nées de pratiques et, parfois, de revendications de groupes et communautés au niveau local. On peut citer à titre d'exemple le cas de la Fédération des totems occitans et catalans (Totemic) née en 2017 de la volonté de protéger ce patrimoine immatériel, « de mener des actions de valorisation des pratiques liées aux animaux totémiques et de trouver, selon ses membres, de "nouvelles formes de gouvernance" " (Storari 2020 : 71).

5 Ainsi pourrait-on avancer que le patrimoine est une des modalités des communs, tant les parentés sont ou du moins semblent nombreuses. Et pourtant les liens entre communs et patrimoine sont finalement largement inexploités. Tout se passe comme si on postulait ce lien sans toujours le questionner véritablement. Il en est ainsi du côté des travaux en sciences du patrimoine, y compris dans des champs dans lesquels les rapprochements seraient les plus évidents. Par exemple, dans les études sur le patrimoine culturel immatériel (PCI), la grammaire des communs semble relativement absente pour qualifier un système de patrimonialisation, dont on peut pourtant dire qu'il est assez proche des figures ostromiennes selon lesquelles le commun se définit ici autour d'une ressource gérée par une communauté - il n'y pas de commun sans communauté - selon certaines règles d'organisation. Ces ingrédients ne se retrouventils pas dans le patrimoine immatériel ? Car ce qui le caractérise, c'est en règle générale une communauté réunie autour d'un intérêt collectif et l'importance capitale dévolue à la participation, donc à la gouvernance, même si sa formalisation n'est guère pensée en tant que telle. Le patrimoine immatériel tout comme les communs est une certaine forme de construction sociale, une forme de recomposition du social, initialement pensée par les promoteurs de la Convention comme un lieu de responsabilité collective. Il est en l'occurrence significatif de voir que plusieurs expériences de communs sont aujourd'hui inscrites sur la liste représentative de l'Unesco : les tribunaux de gestion de l'eau, les prudhommies, les pratiques coopératives, etc. On défend ici ces pratiques en activant la catégorie du Patrimoine culturel immatériel (PCI). De ce point de vue, le processus d'inscription aux inventaires nationaux du PCI ou aux listes de l'Unesco mène souvent à fédérer les acteurs concernés, les pousse à affirmer leur intérêt commun et à entreprendre des démarches qui à l'évidence relèvent de ce champ des communs. L'émergence de la notion de patrimoine culturel immatériel, il y a une vingtaine d'années, aurait ainsi conduit à une reconnaissance des communs et au renforcement graduel du rôle des communautés. Mais pour autant, les acteurs impliqués ne se revendiquent à aucun moment d'une telle démarche. La perspective des communs n'est, en règle générale, pas même questionnée ou mise à l'épreuve. Le 
même constat peut être dressé dans les travaux sur le patrimoine matériel, alors qu'il y aurait sans doute un intérêt à ce croisement entre patrimoine et communs. Tout au plus s'accorde-t-on sur l'idée que le patrimoine est un bien commun, une propriété commune, ce que signale la doctrine italienne des «beni comuni» qui y inclut explicitement le patrimoine culturel et historique ${ }^{5}$. Cette analyse est confirmée par Daniela Festa qui, en parcourant la tradition juridique italienne, met en évidence le lien étroit entre ces deux catégories. Cette qualification se traduit sur le plan pratique par la nécessité de garantir un usage collectif et de créer des entités qui soient en mesure d'assurer une gestion inspirée par les principes de participation. Les biens culturels sont ainsi des biens communs dont l'usage collectif doit être ménagé.

Malgré l'évidence de cette proximité entre patrimoine et communs, les écoles de pensée sur les communs se sont en grande majorité déployées en dehors de toute référence au patrimoine. Il est certes un possible objet des communs, mais il n'est que très rarement mobilisé comme catégorie notionnelle susceptible d'alimenter la réflexion sur les communs, si l'on met de côté la théorie du patrimoine comme transpropriation développée par François Ost. Ces deux territoires des communs et du patrimoine semblent bien suivre, chacun, leur route, sans intersections notables.

7 La catégorie de patrimoine suffirait à caractériser la question patrimoniale, à en endosser les enjeux contemporains et finalement, dans cet exercice, la notion de communs ne serait pas d'une grande utilité. L'hypothèse mérite débat. Le mot, déjà, dans sa proximité avec la notion économique et civiliste (les deux acceptions privatiste et publiciste le plus souvent arrimées), produit certains registres de discours très marqués par une approche propriétariste, y compris lorsque les biens sont publics au sens juridique du terme. Or c'est aujourd'hui précisément ce qui est hors propriété, ce que l'on conçoit comme hors de l'emprise d'une maîtrise exclusiviste, que l'on cherche aussi à relier à la figure du patrimoine culturel - une figure du patrimoine qui se rapprocherait de cet «hypercommun» que Diego Landivar appelle de ses vœux. En outre, le patrimoine est très imprégné d'une conception institutionnelle et ce mode de captation évacue tout un pan de réflexion sur les usages et intérêts multiples des choses ainsi qualifiées, sur ce lieu de négociation entre acteurs multiples (l'État, le public, ceux qui ont un droit sur la chose mais aussi ceux qui plus diffusément y ont un intérêt). Le recours au commun pourrait-il alors être une possible clé de réinterprétation du patrimoine?

\section{L'eau comme patrimoine, comme commun et comme politique}

$8 \quad$ La lecture des différentes contributions montre à quel point l'exercice ne tombe pas sous le sens. Tenir ensemble patrimoine et communs est tout sauf évident. Le cas de l'eau, abordé par trois contributions, est révélateur de cette situation. L'eau est un bien commun, « un bien commun par excellence ", s'accordent à dire Elisabetta Cangelosi et Sandrine Simon. Or la première met en exergue toutes les ressources sociales et politiques de l'idée de commun, quand la seconde fait valoir le patrimoine comme une ressource qui renforce et légitime le commun. En effet, la notion de patrimoine est centrale dans l'analyse de Sandrine Simon qui met en évidence l'importance de la redécouverte des savoir-faire traditionnels et des modes de gestion communautaires de l'eau dans les zones arides et semi-arides marocaines, et donc la nécessité d'inclure les 
communautés concernées détentrices de ces savoir-faire dans les nouveaux modes de développement. L'eau renvoie à l'ensemble des "savoir-faire traditionnels reconnus comme particulièrement bien adaptés aux milieux naturels et aux structures sociales ». Cette redécouverte impulse une réorientation de la politique de l'eau au Maroc, présentée comme désormais fondée sur la conjugaison de l'eau comme bien commun et de l'eau comme matière à patrimoine(s) immatériel(s). Le lien patrimoine et commun tient ici au fait que le patrimoine enrichit et concrétise la pratique du (bien) commun dans la mesure où elle met l'accent sur la centralité des acteurs concernés, véhicule des expériences, des modes de gouvernance, des valeurs qui contribuent à construire le collectif qui lui est associé. Quant à Elisabetta Cangelosi, elle se focalise sur les principaux évènements qui ont fait émerger la notion d'«eau bien commun de l'humanité ». La "guerre de l'eau» de Cochabamba ou encore le Forum italien pour l'eau s'opposent à l'idée que l'eau puisse être considérée comme une marchandise et mettent en évidence le lien étroit entre communs et droits humains. Les biens communs sont alors abordés comme outils de transformation sociale, et «l'action sociale et politique autour de l'eau comme bien commun contribue à créer une nouvelle idée de public et un modèle alternatif de société ». De fait, on observe un glissement d'une réflexion en termes de biens communs naturels à une réflexion plus politique en termes de commoning et si l'auteure évoque la possibilité de repenser la notion de patrimoine à partir de là, elle montre, peut-être sans le vouloir, qu'on peut très bien s'en passer.

9 On peut dire que Leo Coutellec poursuit cette voie alternative, politiquement située et informée, lorsqu'il rend compte d'une expérience, à laquelle il a activement participé, qui a consisté à convaincre les élus d'une communauté de commune française de ne pas reconduire la gestion de l'eau potable par délégation de service public à une entreprise multinationale privée et de créer une régie publique intercommunale. La rencontre avec le patrimoine n'a pas lieu parce que ce n'est pas une catégorie mobilisée dans ce type d'action qui consiste à reconquérir un commun qui a été confisqué ${ }^{\text {. Comme le }}$ note Elisabetta Cangelosi, «l'utilisation du concept de patrimoine est très rare parmi les activistes ». Et parce que, pourrait-on ajouter, Léo Coutellec se place d'emblée sur le terrain du politique. Considérant qu'il n'y a "pas de commun sans politisation", l'auteur revendique "une mise en politique par le bas, par ses usagers, par la construction d'un rapport communal à l'eau»: «Ce n'est pas seulement produire du discours politique sur un commun, ce n'est pas inscrire ce commun dans des catégories politiques bien installées, c'est le constituer en tant que tel, c'est lui donner sa consistance. » L'eau " ne s'appréhende comme commun que depuis sa réappropriation concrète, matérielle, située, sensible ». Il s'agit moins de se réapproprier un bien que de faire commun à son endroit. Le geste de reprise est autant important que la prise : reprise et prise se codéterminent et sont dès lors interdépendants : un bien commun, dans cette perspective, est un faire commun.

Léo Coutellec livre une leçon de politique qui trace son chemin en se gardant des écueils de l'apolitisme (s'en remettre aux experts) et de la polarisation (confondre la lutte, concrète et située, et sa théorisation) et ouvre « une brèche d'émancipation ». Et sans doute faut-il alors interroger la tonalité du politique en question. Même non polarisée, la démarche engagée est instruite ou du moins sous-tendue par une perspective d'écologie politique anticapitaliste, dans la voie tracée, pour le dire vite, par André Gorz ${ }^{7}$. Elisabetta Cangelosi situe son propos dans une orientation similaire 
lorsqu'elle fait valoir que la construction de l'eau comme «bien commun public » se dresse contre sa marchandisation induite par les politiques néolibérales. Les deux auteurs se placent en rupture avec le paradigme de l'extractivisme (Bednik 2016), inhérent à la modernité capitaliste et consistant dans l'exploitation maximale ou maximisée des ressources naturelles. Rompre avec l'extractivisme, c'est rompre avec une logique d'action centrée sur la seule ressource. Sans évoquer ce paradigme, Sandrine Simon met sous le signe d'un aménagement ou d'une atténuation de la raison extractiviste au nom du développement durable le déplacement qu'elle observe, de la surexploitation privée de l'eau à grands renforts technologiques - des barrages et des forages - peu soucieuse de l'environnement comme des populations locales, à sa recommunisation. De ce point de vue, il est significatif qu'elle conclue son propos en avançant que la redécouverte du patrimoine de l'eau - i.e. de l'eau comme patrimoine laisse augurer, après une modernité niveleuse et destructrice de biens communs et de patrimoine, une entrée dans une postmodernité qui serait fondée sur l'alliance « du patrimoine culturel immatériel et de nouvelles technologies » et qui permettrait de "réconcilier société civile et gouvernement». La corrélation ainsi faite, ici, entre patrimoine, paix sociale et croissance post-moderne comme l'absence, là, de l'invocation de la raison patrimoniale quand il s'agit de jeter les bases d'un monde alternatif ne doivent pas manquer de nous interroger sur les significations à inférer de la rencontre du patrimoine et des (biens) communs.

\section{Le patrimoine, des biens communs au commoning}

11 Le détour par ces approches de l'eau-comme-bien-commun permet de dégager une piste singulière pour penser le patrimoine aujourd'hui à la lumière ou plutôt sous la pression du paradigme des communs et de sa portée potentiellement transformatrice. Les lectures des autres contributions vont nous permettre de l'affiner et de la nuancer, en particulier dans un glissement progressif vers le commoning.

12 À l'instar de Sandrine Simon, Enrico Bertacchini vise la référence à ce qu'il appelle « un nouveau paradigme de développement»: c'est-à-dire un développement local, équitable et durable. Dans cette perspective, il cherche à définir un cadre à la fois théorique et pratique qui permette de promouvoir une analyse économique appliquée au domaine de la culture et du patrimoine qui soit appropriée à ce nouveau paradigme. Il s'inscrit pour cela dans la veine ouverte par le rapport de l'ONU, Notre diversité créatrice (Perez de Cuéllar 1996), qui, à la fin du siècle dernier, a cristallisé et déployé un débat qui a eu son importance sur la place de la culture dans le schéma directeur du développement durable ${ }^{8}$. Il voit dans la perspective des biens communs ouverte par Elinor Ostrom la possibilité d'enrichir l'analyse économique du patrimoine culturel et de résoudre les problèmes que posent sa conservation et sa gestion. Pour cela, il propose une approche intégrée permettant de définir le rôle du patrimoine dans le développement durable, appuyée sur trois concepts clés combinés et interagissants : le capital culturel - le patrimoine est un actif immobilisé produisant d'autres biens et services, il s'ensuit que les valeurs culturelles qu'il incarne sont mises sur le même plan que les valeurs économiques qu'il peut générer - ; le district culturel, qui fait valoir la dimension entrepreneuriale, organisationnelle et territoriale de la production de biens et de services liés aux biens patrimoniaux; les biens communs culturels, qui ouvrent sur la prise en compte des communautés locales et questionnent la gouvernance des 
diverses dimensions du patrimoine en tant que ressource partagée. Si l'on reprend la partition sensiblement esquissée à propos de l'eau entre manières de "faire monde ", on notera que d'un point de vue réellement ou radicalement alternatif, la notion de développement durable n'est pas ou plus tenable quand elle n'est pas considérée comme dépassée au regard de l'état de dévastation de la planète et des perspective sombres quant à son habitabilité 9 . Par contraste, la proposition de l'auteur, qui relève du marketing et des sciences économiques et de gestion, s'inscrit dans une perspective aménagementiste $^{10}$ et, s'agissant du développement territorial il ne serait pas inintéressant de la confronter au projet théorisé par Alberto Magnaghi (2014) de "retour au territoire comme bien commun", sous l'appellation de "biorégion urbaine ».

13 À travers une interrogation sur les notions de communauté et de participation, deux notions censément phares de la Convention pour la sauvegarde du PCI, Cléa Hance tente de cerner les collectifs, différemment nommés, susceptibles d'être concernés : peuple, minorité ou groupe, peuples autochtones, tribaux, indigènes, migrants..., et d'évaluer la possibilité que leurs intérêts puissent faire l'objet d'une reconnaissance juridique, sous la désignation "d'intérêt commun des communautés ». On sait que la notion de communauté n'est guère retenue par le système juridico-administratif français et qu'elle ne doit de l'être (faiblement) qu'à la faveur de la ratification de la Convention par la France. C'est précisément le rôle que se sont donné des institutions internationales telle l'Unesco, par l'entremise d'instruments culturels et patrimoniaux, que de "protéger" sous cette désignation tout collectif qui ne bénéficie pas de l'identification à une communauté nationale. L'apport de la Convention de 2003 serait de taille puisqu'elle consacre la subjectivité du bien commun distingué et fait du caractère commun la source d'un droit de participation. Or, au niveau du droit international, cette reconnaissance bute sur deux types de résistances: d'une part, la difficulté de faire valoir en pratique le caractère collectif du droit de participation; d'autre part, celle de s'affranchir du prisme libéral en tant qu'il sacralise la protection des droits individuels et perçoit « la reconnaissance du droit des communautés comme une menace pour l'exercice des libertés politiques ». Cléa Hance veut cependant croire que la reconnaissance du PCI, de droits environnementaux, de modes de vie traditionnels permet de revendiquer un intérêt commun indivisible, c'est-à-dire qui n'est pas la somme des intérêts individuels. Reste à apprécier la portée pratique de cette brèche dans la raison libérale.

Présentant la situation italienne, Daniela Festa esquisse un autre chemin que celui emprunté par Enrico Bertacchini, tout en creusant aussi le dialogue possible entre commun(s) et patrimoine. Elle part de ce que fait la fonction sociale du patrimoine au droit pour comprendre l'association faite aujourd'hui, de plus en plus, entre biens culturels et biens communs ou parfois communs tout court. Elle estime que ces références "restent souvent floues et peu développées". "Pouvons-nous ainsi considérer les biens culturels en tant que biens communs ou communs? Peut-on interpréter la relation à ces biens sous l'angle de l'inclusivité ${ }^{11}$ et non pas de l'exclusivisme propriétaire et dans quel sens?" S'appuyant en particulier sur les travaux de François Ost, et sur la notion de «transpropriation » (Ost 2003) que celui-ci applique aux biens environnementaux en tant que "patrimoine commun", elle en vient à superposer les deux notions de patrimoine et de bien culturel, en les plaçant en particulier à l'enseigne du paysage et du territoire - du paysage comme territoire. C'est là sans doute qu'il lui importe de faire la distinction entre bien commun et commun 
pour envisager que la catégorie des communs puisse aider à retravailler cet objet patrimoine, être non seulement un lieu de questionnement de son degré de communalisation, mais aussi plus radicalement un lieu de révélation et de transformation de la fonction sociale du patrimoine. Les communs pris comme ressource heuristique font voir où en sont les failles ${ }^{12}$. Le détour par les communs montre à quel point nos systèmes s'inscrivent dans des schémas de pensée finalement assez réducteurs ordonnés autour du pouvoir de contrainte de l'État, raisonnés en termes de souveraineté. Les multiples intérêts qui s'expriment sur un bien culturel n'ont ainsi guère de réalité juridique ou politique. Daniela Festa veut interroger la solidité de cette théorie émergente des communs et sa capacité à améliorer les analyses et solutions formulées " pour répondre aux questions de notre temps ». Aussi n'est-on pas étonné qu'elle conclue son article par une échappée dans « le commoning des biens culturels urbains ", soit des expériences, à Rome, à Naples et à Turin, de réappropriation, avec l'assentiment ou non des pouvoirs publics, des espaces urbains par des «travailleurs de l'immatériel », des précaires de la recherche, de l'art et de la culture. Ces expériences, «socialement génératives », conférant aux lieux investis leur double dimension de patrimoine culturel et de fabrique de l'immatériel émargent au phénomène des friches culturelles, mais ne sont pas également sans évoquer aussi les formes de "patrimoine insurgent " que Leonardo Barci Castriota et Gabriel Braga (2016) décrivent dans le Brésil contemporain: des occupations de lieux porteurs d'histoires qui s'inscrivent dans une dynamique de mouvements sociaux d'action directe qui veulent prendre le contrepied du processus de plus en plus insistant de marchandisation du patrimoine, soutenu et encouragé par les politiques néolibérales mises en œuvre par l'État et qui livrent les villes aux investisseurs privés.

La marchandisation est au cœur de l'analyse de Meryem Deffairi, qui la pose en tant que problème mais avec un accent nettement plus pessimiste. S'intéressant au phénomène de patrimonialisation de l'environnement dans le prolongement de ses travaux de thèse (Deffairi 2015), elle considère que le concept de " patrimoine commun [...] est le plus à même d'incarner la richesse et la complexité des travaux sur la gouvernance et les usages menés par les politistes, économistes et philosophes au sujet des communs ». La notion, portée par le droit de l'environnement pourrait être, selon elle, tout à la fois le bon « vecteur de la protection des biens environnementaux et le meilleur biais de réception de la théorie des communs». Mettant en exergue la veine solidariste de ce concept, dégagée notamment par Jérôme Attard (2003), elle suggère que communs et patrimoine sont deux notions sœurs, mais pointe tout en même temps la faible armature conceptuelle et opérationnelle de celle de patrimoine commun, qui reste largement indéterminée ${ }^{13}$, et les effets de résistance dans le droit et dans les discours sur le droit. Le phénomène de patrimonialisation, dans le droit de l'environnement, s'éloigne en l'occurrence parfois singulièrement de la logique des communs avec le recours à des instruments juridiques qui relèvent de mécanismes de marché. "Alors que les ressources naturelles sont rattachées par le législateur à un "patrimoine commun", compris comme une richesse collective à préserver, elles sont, en parallèle, l'objet de droits patrimoniaux privatifs ${ }^{14}$ de plus en plus importants et d'une commercialité juridique, voire économique témoignant d'une appréhension croissante des ressources naturelles par le marché, allant a priori à contresens de leur rattachement à la catégorie des "communs" impliquant la mise en relief de droits d'usage collectifs spécifiques, voire d'une forme de propriété commune». Une piste esquissée pour résister à ces logiques de marchandisation est que "les travaux 
consacrés aux communs enrichiront à l'avenir les réflexions des juristes ». Mais en réalité communs et patrimoine ne sont guère investis ensemble. Et on le conçoit volontiers tant, comme l'observe Meryem Deffairi, «le relais de ces travaux (sur les communs) n'est pas toujours assuré par la doctrine juridique et par le droit positif pour lesquels le carcan propriétariste mais également les modèles traditionnels d'action publique demeurent difficiles à abandonner ».

Elisabetta Cangelosi interroge, dans une deuxième contribution, les relations entre (biens) communs, droits des femmes et justice de genre : il s'agit de mettre en exergue le rôle des femmes dans la création et la protection des communs et de montrer l'importance de la perspective de genre pour étudier le lien entre les biens communs et le patrimoine immatériel. Ce n'est a priori pas évident dans la mesure où, même si elles prennent une part significative dans les batailles pour les biens communs et contre l'accaparement et la marchandisation des terres, "les revendications relatives aux droits des femmes n'y sont pas explicitement incluses ». On touche ici à l'ambiguïté de la référence à la tradition, dans les sociétés paysannes ou autochtones, qui n'épouse pas, loin s'en faut, la cause des femmes. Aussi l'auteure se demande à juste titre ce que, en retour de leur rôle, ces batailles pour le droit à la terre, à la ville, au logement, etc. font pour leurs droits et la justice de genre. Précisément, elle voit dans la pratique du commoning, "avec ses caractéristiques de réinvention et de transformation de l'imaginaire (et de la réalité quotidienne des communautés) » " un terrain fertile pour ces revendications ». Si «les femmes émergent comme actrices et bénéficiaires de la réinvention, de la création et de la protection des biens communs ", c'est sans doute au regard d'un contexte général de plus en plus insistant de justice climatique et environnementale.

\section{Gestes spéculatifs}

17 Enfin, deux contributions, celle d'Alessia Tanas et Serge Gutwirth et celle de Diego Landivar, proposent de penser les approches en termes de commun(s) et de patrimoine comme contribuant à la transformation des modes de vie et d'habiter sur de nouveaux fondements juridiques, politiques et économiques. Elles se distinguent en ce qu'elles relèvent du "geste spéculatif ", quand on entend par cette notion, à la suite de Didier Debaise et Isabelle Stengers (2015: 4), la capacité que l'on se donne d'engager «la pensée au service de possibles qu'il s'agit d'activer et de rendre perceptibles dans le présent ». Se référant à des situations passées ou présentes, ces possibles insistent. Il appartient à la pensée spéculative, nourrie de récits et de narrations qui projette dans le futur incertain, soucieuse de ne rien exclure et de ne rien disqualifier, de les faire importer, d'en intensifier le sens (Debaise \& Stengers 2016).

Geste spéculatif quand Alessia Tanas et Serge Gutwirth s'attachent à relier trois figures représentatives du paradigme des communs, le patrimoine transpropriatif, les droits d'usage dits "usi civici» (usages civique) italiens et la rivière-personne, et en font la matrice d'une pensée jurisprudentielle et d'une approche qu'ils nomment " écologique ". Pour cela, ils placent explicitement leur référence à la résurgence des communs sous le signe du commoning. Selon leur définition, le commoning tient à quatre éléments : «Il y a commoning quand /1/ des personnes /2/ s'auto-organisent /3/ autour d'une « chose » qui les concerne et les responsabilise collectivement, /4/ et poursuivent des activités marquées par leur générativité plutôt que par l'extraction ${ }^{15}$.» Cette 
définition est la marque de leur approche, en ce qu'elle veut être distinguée d'une focalisation sur les ressources comme d'une focalisation sur les communautés de personnes et les modes d'organisation. Si elle adhère pleinement à la maxime selon laquelle "there is no commons without "commoning"16", signifiant que s' « il n'y a pas de communs sans action commune, un commun n'existe qu'au gré de l'action qui le produit et le génère » - maxime qui, faut-il le noter, résume également la démarche de Léo Coutellec-, elle met l'accent sur les liens « sympoïétiques ${ }^{17}$ » qui caractérisent un collectif vivant, qui assurent l'interconnectivité entre des communautés et leur territoire, des relations de réciprocité et d'interdépendance entre toutes les formes de vie, humaines et non humaines, et les choses qui le composent. Constatant la faible réception par le droit droit de ce "genre d'agir durable et génératif", même s'ils observent que «la législation, la doctrine et la pratique juridique ne sont pas complètement insensibles aux interpellations et démarches (pro)posées par 㕆 ces医 modes de vie et d'habitation du monde», ils proposent une voie qui déjoue "l'alternative infernale et toxique » d'un agir qui n'aurait d'autres solutions que d'en passer par le marché ou bien de s'en remettre à l'État et qui vise à faire émerger des modalités de prise en compte et de protection des rapports qui constituent en euxmêmes des ensembles vivants. D'où l'importance du thème de la résurgence : ce qui fait retour n'est pas un retour en arrière, mais s'installe dans le présent et pour le possible comme ce qui a été éradiqué et ne demande qu'à reprendre son cours pour d'autres mondes à fair $e^{18}$. Usages multiples pour une multiplicité des titulaires, droits séculaires d'usage agricoles, sylvicoles et pastoraux, institution d'un fleuve comme personne juridique, ces conceptions, pratiques et situations portent toutes une remise en question du régime propriétariste et sont susceptibles de trouver une résolution juridique dans la théorie de la chose-milieu élaborée par Sarah Vanuxem (2018). Les auteur'e's concluent sur le constat qu'il n'est cependant pas possible de faire coexister tous les intérêts - i.e. toutes les manières d'habiter la chose -, quand leur démarche se veut spéculative, au sens où elle invite à «ne pas exclure ou à disqualifier mais à penser ce qui vient ». Il reste en conséquence à se débarrasser d'intérêts qui disqualifient, de ceux qui ne se satisfont qu'au moyen de confiscations et d'éradications.

Geste spéculatif quand Diego Landivar suggère de livrer bataille et de dresser des barricades ontologiques devant la force ontologique majeure, le "geontopower » qui, selon Elizabeth Povinelli (2016), caractérise le mode opératoire du libéralisme tardif, soit par re-catégorisation et distribution des êtres et les chose qui le composent sur un axe vie-non-vie, décret d'espace vides, pour satisfaire à ses fins extractivistes et marchandes. Pour cela, il propose de rapprocher trois opérations politiques, l'animisme juridique inspiré des cosmologies amérindiennes, le patrimoine comme héritage et la philosophie des communs. S'il reconnaît qu'elles ne relèvent pas d'une filiation épistémique commune ${ }^{19}$, il avance que, face à l'urgence climatique et écologique, elles dessinent de nouveaux horizons politiques pour notre époque, en tant que modes de contestations ontologiques capables d'intensifier des existences, objets, artefacts, territoires, entités non humaines, divinités, de les faire importer, de les redignifier, et donc de résister au geontopower. Faire du patrimoine une scène cosmopolitique où se négocie, entre humains et non-humains, ce à quoi on tient et ce à quoi on renonce, radicaliser le geste protecteur de la philosophie des communs par focalisation sur le commoning, "activité commune en train de se faire", et par institution de "commeun "- des espaces travaillés par des alliances créant « des continuités politiques entre humains et non-humains»-, engager des «inventions cosmologiques [bien plus 
qu'actualiser des cosmologies autochtones] enracinées dans des opérations diplomatiques contemporaines " visant à "faire advenir de nouvelles fictions légales " (telles que la rivière-personne ou la Pachamama), les opérations mobilisées par Diego Landivar sont poussées au plus fort de leur radicalité : du patrimoine comme opération de maintien (d'un ordre dutemps) au patrimoine comme recomposition des attachements, des biens communs au commoning, de la capacité incorporée dans le droit de mettre des entités hors du commerce à la constitution d'une arène où des humains de tous bords composent avec une rivière. La force des barricades est à ce prix, mais il va plus loin en rappelant que le geontopower agit comme force colonisatrice qui détourne et enrôle au service du libéralisme tardif, ce qui revient à dire, pragmatisme oblige, que les opérations par lesquelles se dressent des barricades sont ambivalentes : le patrimoine est aussi - et peut-être surtout - une ressource pour une économie de l'enrichissement ; le capitalisme sait faire fond sur les résurgences animistes telles que le néo-chamanisme. "Le Capitalisme, écrit Elizabeth Povinelli, a une relation unique avec le Désert, l'Animiste et le Virus ${ }^{20}$ dans la mesure où le Capitalisme voit toutes choses comme ayant le potentiel de créer du profit; c'est-à-dire que rien n'est intrinsèquement inerte, tout est vital du point de vue de la capitalisation, et tout peut devenir quelque chose de plus avec le bon angle d'innovation. Au vrai, on peut dire que les capitalistes sont les plus purs des Animistes » (Povinelli 2016:20, notre traduction). Autant dire la nécessité, souligne l'auteur, de ne pas attaquer le libéralisme tardif sur son terrain.

\section{Quel patrimoine?}

Il est frappant de constater que c'est dans le domaine de l'« environnement ", ou plutôt de l'écologie politique, que naît ou renaît la doctrine contemporaine des communs et que tout en même temps se renouvelle la notion de patrimoine réappropriée par le Code de l'environnement pour désigner tout élément de la biodiversité. L'argument patrimonial est mis en avant dans la caractérisation des dommages considérables causés par le naufrage de l'Erika ${ }^{21}$. Les deux notions de patrimoine et de communs, censément, luttent contre les mêmes fléaux : les désastres écologiques, la faillite des modèles institutionnels, la crise de l'idéologie propriétaire ${ }^{22}$, avec chacun, ses outils conceptuels. Chacun les affûte isolément, alors même que ces mouvements ont en commun de s'attacher à ce que nous devons sauver, mais aussi «à ce à quoi nous sommes prêts à renoncer ». La réflexion proposée par Diego Landivar permet ainsi d'aborder les communs non seulement du point de vue des activités de régulation, "mais aussi des opérations de revendication (ontological reclaims) face à un aplatissement ontologique du monde ».

Elle permet de poser de façon plus décisive la signification du patrimoine lorsqu'il rencontre les communs. Car on ne peut pas limiter son apport à l'élaboration d'un droit de l'environnement. De ce point de vue tou'te's les auteure's de ce numéro sont amenée's à considérer l'étendue du mot et toutes les catégories d'objets, de pratiques et d'êtres qu'il englobe. Au plan juridique, la notion de transpropriation, justement citée par plusieurs contributeur-rice's, semble la plus féconde pour donner aux objets un statut tel qu'ils ne soient pas irréductiblement enfermés dans le régime de la possession. Elle contribue à faire bouger la notion de patrimoine, comme ont tenté de le faire, avec des fortunes diverses, des instruments internationaux tels que la Convention 
Unesco de 2003 ou la Convention de Faro. Mais est-ce suffisant? Les auteurees qui observent le glissement des biens communs vers les communs sont de fait amenée's à nuancer la place et l'apport du patrimoine, à moins de l'envisager dans une optique qui le dévie nettement de ses rails institutionnels. Pour justifier son usage du patrimoine comme barricade ontologique, Diego Landivar a recours à l'analyse du patrimoine que propose l'un de nous : qu'en est-il de l'opérativité du patrimoine à ou «devant» l'anthropocène (Tornatore 2017)? Question poursuivie par une interrogation sur la possibilité d'écrire un scénario contre-hégémonique du patrimoine (Barbe 2019; Tornatore 2019, 2020). Contre-hégémonique car cela suppose de se démarquer du mainstream patrimonial tel qu'il est représenté par et composé des politiques et des économies du patrimoine : en d'autres termes, le patrimoine comme instrument d'instauration des États-nations, de contrôle des individus et d'aménagement des territoires, comme actif de développement, comme agent de croissance et d'enrichissement. Loin des analyses étouffantes et dépolitisées en termes d'identité et de pérennité, il s'agit dès lors de prendre la mesure de sa portée au regard des enjeux de notre siècle devant les perspectives d'effondrement, de sa signification comme héritage, alors que nous héritons d'un monde ravagé et en ruine, comme attention, alors que le capitalisme s'est arrogé le droit de ne pas faire attention (Stengers 2008), comme attachement et interdépendance, alors que le libéralisme veut des individus libres et sans attaches, comme inséparation, alors que nos conceptions du monde sont bâties sur les solides dualismes du sujet et de l'objet, de la nature et de la culture, du corps et de l'esprit, comme participation, alors que toute politique, si locale soit-elle, fonctionne sur la désignation et la délégation d'experts...

En bref, ni l'État ni le marché, alors quoi ? Faut-il, pour trouver le patrimoine, se défaire du besoin que nous avons des institutions patrimoniales ? Il importe, et c'est sans doute l'aboutissement (provisoire) du cheminement de ce numéro, de mesurer l'apport du paradigme des communs et des institutions qu'il génère et sur lesquelles il repose. On ne peut ignorer les raisons qui ont conduit à l'essor des communs. Elles sont dites ici explicitement ou en creux. Revenir sur la pseudo-tragédie des communs, c'est être au moins conduit à interroger sinon à remettre en cause les fondements juridiques, politiques et économiques qui assoient le monde ayant pour nom modernité à grands coups de disqualification, d'éradication, d'enclosures, de dépossession, de prise (de terres), de partitions, de segmentations, de marketing... qui conditionnent humains et non-humains, le vivant comme le non-vivant, et qui aujourd'hui se réalisent dans le geontopower du libéralisme tardif. Pour peu que l'on renonce au développement et à la croissance - le lecteur se fera sa religion -, pour trouver le patrimoine, il faut suivre la voie des communs, plus précisément la voie du commoning - quitte au bout du compte à ce que le patrimoine s'y dilue, renvoyant le temps du patrimoine dans l'histoire des sociétés occidentales et cédant la place à un nouveau temps, celui des communs. Les fondements sur lesquels est construite la structure de signification «moderne » du patrimoine vacillent: les communs ébranlent le socle juridique. Ils ne sont pas sans liens avec d'autres socles qui également se fissurent et qui ont au trait au temps, au politique, et au savoir ${ }^{23}$.

23 À suivre... 


\section{BIBLIOGRAPHIE}

ATTARD Jérôme, 2003, «Le fondement solidariste du concept "environnement-patrimoine commun" ", Revue juridique de l'environnement, $\mathrm{n}^{\circ}$ 2, p. 161-176. Disponible en ligne, https:// www.persee.fr/doc/rjenv_0397-0299_2003_num2_4146 [lien valide en mars 2021].

BARBE Noël, 2019, « Pour une anthropologie plébéienne et pragmatiste du patrimoine. En finir avec le patrimoine patricien. Par le bas et par la marge », In Situ. Au regard des sciences sociales, $\mathrm{n}^{\circ} 1$, « Le tournant patrimonial » [en ligne], http://journals.openedition.org/insituarss/485 [lien valide en mars 2021].

BEDNIK Anna, 2016, Extractivisme. Exploitation industrielle de la nature : logiques, conséquences, résistances, Neuvy-en-Champagne, Le Passager clandestin.

BENASAYAG Miguel \& COUTELLEC Léo, 2018, « Nos limites ne sont pas les leurs. De la nécessité d'une approche critique de la notion de limite ", Écologie \& politique, ${ }^{\circ}$ 57, vol. 2, « Retour sur terre. Pour une éthique de l'appartenance », p. 117-132. Disponible en ligne, https:// www.cairn.info/revue-ecologie-et-politique-2018-2-page-117.htm [lien valide en mars 2021].

BOLLIER David, 2014, Think like a commoner. A short introduction to the life of the commons, Gabriola Island, New Society Publishers.

CASTRIOTA Leonardo Barci \& BRAGA Gabriel, 2016, « Patrimônio insurgente: estetização e resistência cultural no Brasil do inicio do Século XXI », communication au congrès international «Contested_cities» (Madrid, 4-7 juillet 2016).

CORIAT Benjamin, 2015, Le Retour des communs. La crise de l'idéologie propriétaire, Paris, Les liens qui libèrent.

CORIAT Benjamin, 2017, « Commun, biens communs, approche économique » [notice], in CORNU Marie, ORSI Fabienne \& ROCHFELD Judith (dir.), Dictionnaire des biens communs, Paris, PUF, coll. « Quadrige».

CORNU Marie, ORSI Fabienne \& ROCHFELD Judith (dir.), 2017, Dictionnaire des biens communs, Paris, PUF, coll. « Quadrige ».

DARDOT Pierre \& LAVAL Christian, 2010, « Du public au commun », Revue du MAUSS, n 35, vol. 1, p. 111-122. Disponible en ligne, https://www.cairn.info/revue-du-mauss-2010-1-page-111.htm [lien valide en mars 2021].

DARDOT Pierre \& LAVAL Christian, 2014, Commun. Essai sur la révolution au XXI siècle. Paris, La Découverte.

DARDOT Pierre \& LAVAL Christian, 2017, « Commun » [notice], in CORNU Marie, ORSI Fabienne \& ROCHFELD Judith (dir.), Dictionnaire des biens communs, Paris, PUF, coll. « Quadrige ».

DEBAISE Didier \& STENGERS Isabelle (dir.), 2015, Gestes spéculatifs, actes du colloque éponyme (Cerisy-la-Salle, 28 juin-5 juillet 2013), Dijon, Les Presses du réel.

DEBAISE Didier \& STENGERS Isabelle, 2016, «L'insistance des possibles. Pour un pragmatisme spéculatif », Multitudes, n 65, " Matières pensantes », p. 82-89. Disponible en ligne, https:// www.multitudes.net/linsistance-des-possibles-pour-un-pragmatisme-speculatif/ [lien valide en mars 2021].

DEFFAIRI Meryem, 2015, La Patrimonialisation en droit de l'environnement, IRJS éditions, coll. « Bibliothèque de l'Institut de recherche juridique de la Sorbonne - André-Tunc ». 
DUSOLLIER Séverine, 2013, «The commons as a reverse intellectual property: from exclusivity to inclusivity ", in HOWE Helena R. \& GRIFFITHS Jonathan (dir.), Concepts of property in intellectual property law, Cambridge, Cambridge University Press, p. 258-281.

DUSOLLIER Séverine, 2015, «Inclusivity in intellectual property », in DINWOODIE Graeme B. (dir.), Intellectual property and general legal principles. Is IP a Lex Specialis?, Northampton (Massachussetts), Edward Elgar publishings.

FELLI Romain, 2008, Les Deux Âmes de l'écologie. Une critique du développement durable, Paris, L'Harmattan, coll. «Biologie, écologie, agronomie ».

FESTA Daniela \& CHARBONNIER Pierre (dir.), 2016, « L'Italie des biens communs » [dossier], Tracés, $n^{\circ} 16$, hors-série, « Traduire et introduire » [en ligne], https://journals.openedition.org/ traces/6509 [lien valide en mars 2021].

FESTA Daniela, 2016, « Les communs urbains. L'invention du commun », Tracés, $\mathrm{n}^{\circ}$ 16, hors-série, «Traduire et introduire» [en ligne], http://traces.revues.org/6636 [lien valide en mars 2021].

GUTWIRTH Serge \& STENGERS Isabelle, 2016, «Théorie du droit. Le droit à l'épreuve de la résurgence des commons », Revue juridique de l'environnement, $\mathrm{n}^{\circ}$ 2, vol. 41, p. 306-343.

HARAWAY Donna J., 2020 [2016], Vivre avec le trouble, Vaulx-en-Velin, Les Éditions des Mondes à faire.

LE CROSNIER Hervé, 2015, En communs. Une introduction aux communs de la connaissance, Caen, C\&F éditions, coll. «Blogollection».

LUCAS Jean-Michel [alias Doc Kasimir BISOU], 2011, Culture et développement durable. Il est temps d'organiser la palabre..., Paris, Irma (Centre d'information et de ressources pour les musiques actuelles), coll. « Révolutic».

MACPHERSON Crawford Brough, 1962, The Political Theory of possessive individualism. Hobbes to Locke, Oxford, Clarendon Press.

MAGNAGHI Alberto, 2014, La Biorégion urbaine. Petit traité sur le territoire, bien commun, Paris, Eterotopia France, coll. « Rhizome ».

MATTEI Ugo, 2011, Beni comuni. Un manifesto, Rome / Bari, Laterza.

MATTEI Ugo, REVIGLIO Edoardo \& RODOTÀ Stefano, 2010, I Beni pubblici. Dal governo democratico dell'economia alla riforma del codice civile, Accademia Nazionale dei Lincei, coll. « Contributi del Centro linceo interdisciplinare Beniamino-Segre ».

NICOLAS-LE STRAT Pascal, 2016, Le Travail du commun, Saint-Germain-sur-Ille, Éditions du Commun.

OST François, 2003 [1995], La Nature hors-la-loi. L'écologie à l'épreuve du droit, Paris, La Découverte, coll. « La Découverte-poche », série « Sciences humaines et sociales ».

PEREZ DE CUÉLLAR Javier (dir.), 1996, Notre diversité créatrice. Rapport de la Commission mondiale de la culture et du développement, Paris, Éditions de l'Unesco.

POVINELLI Elizabeth A., 2016, Geontologies. A requiem to late liberalism, Durham, Duke University Press.

ROCHFELD Judith, 2014, "Quel modèle pour construire des communs? », in PARANCE Béatrice \& SAINT-VICTOR Jacques de (dir.), Repenser les biens communs, Paris, CNRS éditions., p. 103-128. 
ROCHFELD Judith, 2017, « Propriété inclusive ou inclusivité » [notice], in CORNU Marie, ORSI Fabienne \& ROCHFELD Judith (dir.), Dictionnaire des biens communs, Paris, PUF, coll. « Quadrige », p. 983-987.

ROCHFELD Judith, 2020, «L'échelle de communalité. Propositions de réformes pour intégrer les biens communs en droit », rapport à la Mission de recherche Droit \& Justice.

RODOTÀ Stefano, 2013, Il Terribile diritto. Studi sulla proprietà private e i beni comuni, Bologne, Il Mulino, coll. « Collezione di testi e di studi ».

SETTIS Salvatore, 2002, Italia S.p.A. L'assalto al patrimonio culturale, Turin, Einaudi, coll. « Piccola biblioteca Einaudi [nuova serie]».

STENGERS Isabelle, 2008, Au temps des catastrophes. Résister à la barbarie qui vient, Paris, Les Empêcheurs de penser en rond / La Découverte.

STORARI Jacopo, 2020, «Dilemme des anthropologues, restriction de la Convention et dynamiques associatives. Premières réflexions sur les "prises" françaises du patrimoine culturel immatériel », in CACHAT Séverine, CHAVE Isabelle \& TORNATORE Jean-Louis (dir.), Patrimoine et territoire. Une parenté conceptuelle en question, Vitré, Maison des cultures du monde / Centre français du patrimoine culturel immatériel, coll. « Cahiers du CFPCI », p. 58-79. Disponible en ligne, https://www.maisondesculturesdumonde.org/patrimoine-et-territoire-une-parenteconceptuelle-en-question [lien valide en mars 2021].

TORNATORE Jean-Louis, 2017, « Patrimoine vivant et contributions citoyennes. Penser le patrimoine "devant" l'Anthropocène ", In Situ. Revue des patrimoines, $\mathrm{n}^{\circ}$ 33, « Patrimoine culturel immatériel et institutions patrimoniales » [en ligne] http://journals.openedition.org/ insitu/15606 [lien valide en mars 2021].

TORNATORE Jean-Louis, 2018 (7 juin), « S'attacher à la ZAD : de quel patrimoine pourrions-nous parler? », lundimatin, $\mathrm{n}^{\circ} 148$ [en ligne] https://lundi.am/S-attacher-a-la-zad-de-quel-patrimoinepourrions-nous-parler-1392 [lien valide en mars 2021].

TORNATORE Jean-Louis, 2019, « Pour une anthropologie pragmatiste et plébéienne du patrimoine : un scénario contre-hégémonique », In Situ. Au regard des sciences sociales, $n^{\circ} 1$, « Le tournant patrimonial » [en ligne], http://journals.openedition.org/insituarss/449 [lien valide en mars 2021].

TORNATORE Jean-Louis, 2020, « Patrimoine et territoire : institution, destitution », in CACHAT Séverine, CHAVE Isabelle \& TORNATORE Jean-Louis (dir.), Patrimoine et territoire. Une parenté conceptuelle en question, Vitré, Maison des cultures du monde / Centre français du patrimoine culturel immatériel, coll. « Cahiers du CFPCI », p. 195-222. Disponible en ligne, https:// www.maisondesculturesdumonde.org/patrimoine-et-territoire-une-parente-conceptuelle-enquestion [lien valide en mars 2021].

TREBULLE François-Guy, 2007, «La propriété à l'épreuve du patrimoine commun : le renouveau du domaine universel ", in BAUDOIN Jean-Louis Baudouin, BÉNABENT Alain, BERGEL Jean-Louis et al., Études offertes au professeur Philippe Malinvaud, Paris, Litec.

VANUXEM Sarah, 2018, La Propriété de la terre, Marseille, Wildproject, coll. « Le monde qui vient ».

WAGENER Noé, 2012, «Personnes publiques et protection du patrimoine. Approche juridique », in KHAZNADAR Chérif, Le Patrimoine, oui, mais quel patrimoine?, Arles / Vitré, Actes Sud / Maison des cultures du monde, coll. «Internationale de l'imaginaire », p. 107-125. 


\section{NOTES}

1. Sur la polysémie de la notion et les différents sens dans lesquels elle est investie, voir Coriat (2017) et Dardot \& Laval (2017).

2. L'expression est de Salvatore Settis (2002), historien de l'art et directeur émérite de la Scuola Normale di Pisa, qui a été un des acteurs les plus actifs dans la défense des biens culturels en tant que beni comuni en Italie.

3. Le lien entre patrimoine et responsabilité est en réalité peu élaboré dans les textes : sur l'idée de les tenir ensemble comme « clés qui permettront de rendre le droit de l'environnement plus effectif et de réaliser les objectifs de protection et de préservation des «biens environnementaux », voir François Ost (2003).

4. À titre d'exemple, la loi CAP, art. 3, affirme que « [1]'État, à travers ses services centraux et déconcentrés, les collectivités territoriales et leurs groupements ainsi que leurs établissements publics définissent et mettent en œuvre, dans le respect des droits culturels énoncés par la Convention de l'Organisation des nations unies pour l'éducation, la science et la culture sur la protection et la promotion de la diversité des expressions culturelles du 20 octobre 2005, une politique de service public construite en concertation avec les acteurs de la création artistique ». 5. Sur cette école de pensée, voir Cornu, Orsi \& Rochfeld (2017 : «Commission Rodotà »).

6. Ici comme à Notre-Dame-des-Landes, voir Jean-Louis Tornatore (2018).

7. Voir Benasayag \& Coutellec (2018).

8. Le débat porte sur le fait de savoir si la culture peut être le quatrième pilier du développement durable - avec le social, l'économique et l'environnemental - ou bien si, comme le soutient JeanMichel Lucas (2011), c'est l'éthique culturelle, fondée sur la reconnaissance de l'universalité des droits culturels, qui subsume les trois piliers et constitue le principe directeur pour un développement durable humain.

9. Les critiques du développement durable depuis une vingtaine d'années sont nombreuses, voir par exemple Romain Felli (2008). Il est patent que cette notion tend à être périmée en tant que guide pour l'action politique, économique, etc. Après avoir été le mantra des décideurs et autres développeurs, elle est désormais supplantée par celle de transition écologique - qui a même son ministère. Pour une approche critique des transitions, voir le carnet de recherche «Penser les transitions » : https://transire.hypotheses.org/.

10. Le mot doit être pris au sens d'aménagement de la croissance ou du capitalisme que l'on trouve dans les expressions « capitalisme vert ", « croissance maîtrisée »...

11. Voir en particulier l'interprétation d'inclusivité proposée par Séverine Dussolier (2015: 104, 110 ; 2013), conjointement à Judith Rochfeld (2017). Pour des références incontournables, voir Macpherson (1962) et dans le même sens Rodotà (2013).

12. Sur cette démarche, biens culturels et biens communs, voir Noé Wagener (2012), perspective développée dans le cadre du rapport rendu par Judith Rochfeld (2020).

13. En dépit de certaines tentatives de construire une véritable catégorie d'action publique. Voir François-Guy Trebulle (2007).

14. Qui forment et renvoient, quant à eux, au patrimoine dans sa conception civiliste.

15. La tension primordiale pour leur approche entre «droit génératif » et "droit extractif » est exposée dans Gutwirth \& Stengers (2016:335) : «Un droit en devenir, inductif, topique, plutôt qu'un droit posé et abstrait, axiomatique et déductif : un droit qui favoriserait jurisprudence et pratiques comme sources, plus que la loi ou/et la "doctrine". »

16. Serge Gutwirth et Isabelle Stengers (2016: 312) préconisent l'emploi de l'anglais « commons» car les termes « commoners » et « commoning » sont difficilement traduisibles.

17. La notion de sympoïèse est très présente dans l'œuvre récente de Donna J. Haraway interconnectée à celle d'Isabelle Stengers. "Sympoïèse, écrit-elle, est un mot simple. Il signifie "construire-avec", "fabriquer-avec", "réaliser-avec". Rien ne se fait tout seul. Rien n'est 
absolument autopoïétique, rien ne s'organise tout seul [...]. Les terriens ne sont jamais seuls [...]. C'est un mot pour caractériser de manière adéquate des systèmes complexes, dynamiques, réactifs, situés, historiques. Un mot pour désigner des mondes qui se forment-avec, en compagnie. La sympoïèse embrasse l'autopoïèse et, de manière générative, elle la déploie et l'étend » (Haraway 2020 : 115).

18. Voir Gutwirth \& Stengers (2016). Sur la mise en regard de la résurgence des communs et de la résurgence autochtone, voir Tornatore (2020: 212 et sq.).

19. On remarquera toutefois la proximité avec les figures mobilisées par Alessia Tanas et Serge Gutwirth qui, quant à eux, les situent dans la mouvance des communs.

20. Les trois figures du geontopower, définies par Elizabeth Povinelli (2016 : chap. 1).

21. CA Paris, 30 mars 2010, n 08/02278.

22. Sur ces phénomènes, à propos des communs de la connaissance, voir Coriat (2015).

23. Voir Jean-Louis Tornatore, «Des cabanes, la transe et la propriété. Éléments pour un scénario contre-hégémonique du patrimoine : vers un temps de communs ", communication au séminaire de l'EHESS «Critical Heritage Studies. Épistémologies, réception et actualité (Europe, Asie, Monde) » (Paris, 15 avril 2019), et au cours du «Midi-causerie » organisé par le Celat-AqàM (Montréal, 14 novembre 2019) (publication en cours).

\section{AUTEURS}

\section{FRANCESCA COMINELLI}

Maître de conférences en sciences économiques à l'université Paris 1 Panthéon-Sorbonne, membre de l'Institut de recherche et d'études supérieures du tourisme (Irest), Équipe interdisciplinaire de recherches sur le tourisme (Eirest, EA 7337)

\section{MARIE CORNU}

Directrice de recherches au CNRS, Institut des sciences sociales du politique (ISP, ENS

Paris Saclay, université Paris-Nanterre, CNRS)

\section{JEAN-LOUIS TORNATORE}

Professeur en anthropologie à l'université de Bourgogne, Laboratoire interdisciplinaire de recherche « Société, sensibilités, soin » (LIR3S, UMR CNRS 7366) 